\title{
Size effect on deformation of duralumin micro-pillars - a dislocation dynamics study
}

\author{
R. Gu ${ }^{\S}$, P.P.S. Leung, A.H.W. Ngan \\ Department of Mechanical Engineering, The University of Hong Kong, \\ Pokfulam Road, Hong Kong, P.R. China \\ ${ }^{\S}$ Corresponding author (email: gurui @hku.hk)
}

\begin{abstract}
:
Two-dimensional dislocation dynamics simulations are used to study precipitate strengthening effects in duralumin micro-pillars. The results show that a refined microstructure may resist and slow down the movement of dislocations inside the confined volume, leading to hardening and weak size dependence of strength. This study illustrates that the deformation behavior of small crystals is controlled by the combined effect of internal length scale and external size of the crystal.
\end{abstract}

\section{Keywords:}

Plastic deformation; dislocation dynamics; size effect; precipitate hardening 
The size effect on the deformation behavior of metallic materials has been intensively studied in recent years. One of the significant findings is that metallic single crystals show a power-law size dependence of strength [1-3], and jerky stress-strain behavior in a stochastic manner [1, 4-9]. With evidences gained from in situ transmission electron microscopy [9, 10] and Laue micro-diffraction $[11,12]$, a number of physical models have been devised to explain the deformation behavior of small crystals. The "dislocation starvation" $[6,13]$ theory considers that mobile dislocations in the small crystal would easily slip through the small crystal without accumulation and multiplication, so that the crystal is constantly maintained in a dislocation-free state. Another group of models considers that the small crystal is not dislocation free initially, but the operation of dislocation sources $[14,15]$ and the interaction of dislocations in a confined environment lacking the mean-field averaging effect [16, 17] may result in the unusual deformation behavior. Another model [18] explicitly predicts that a power-law size dependence of strength stems from Taylor-type interactions in a fractal dislocation network, and that the power-law exponent is inversely related to the fractal dimension of the dislocation network.

In addition to the extrinsic size effect, several recent studies have also explored how the mechanical properties of small crystals are affected by changes in the intrinsic microstructural length scale, such as decreasing the interaction distances between dislocations by trapping them inside the crystal by surface coating [19-22], introducing precipitates [23], grain boundaries [24, 25], and nano-crystalline [26, 27] or nano-twinning [22, 28, 29] microstructures into the crystal. Our previous experimental study [23] on the deformation behavior of duralumin micro-pillars has shown that with the presence of second-phase precipitates, strength is much improved and less size-dependent, and a very high density of dislocation debris is retained in the deformed microstructure suggesting that mobile dislocations are slowed down by viscous drag from the precipitates. In this study, two-dimensional (2D) dislocation dynamics simulations [30-33] are used to study the deformation behavior of these duralumin micro-pillars. The distribution of the precipitate particles and the external size of the specimen are varied to investigate their effects on the plastic properties of the micro-pillars.

In the present 2-D simulations, screw dislocations are considered and they are randomly distributed in a simulation region with a given initial density $\rho_{0}$. The simulation region is rectangular where the short and long sides, in a ratio of 1:3, respectively represent surfaces 
parallel and perpendicular to the radial direction of a micro-pillar. Periodic boundary conditions are applied at the two short sides of the simulation region, which means that as a dislocation escapes from one side it re-enters into the region from the opposite side. The other two long sides are regarded as free surfaces where nearby dislocations would be affected by image forces and may therefore be likely depleted on them. Two orthogonal slip systems are considered in the simulation region, which are oriented with $35.2^{\circ}$ and $125.2^{\circ}$ from the radial direction. The material properties of aluminum are used in this simulation. Dislocations gliding in the slip plane may sustain three types of forces without meeting any precipitates: (i) the pair-wise elastic interaction force $f_{i j}=\frac{\mu b^{2}}{2 \pi r_{i j}}$ between dislocations, where $r_{i j}$ is distance between two interacting dislocations, $\mu=26 \mathrm{GPa}$ is shear modulus and $b=0.3 \mathrm{~nm}$ is the magnitude of the Burgers vector; when a dislocation is moving into a region within a distance of cut-off radius from a free surface, the image force is also worked out by this relation. (ii) A resistance stress opposite to the glide direction due to Taylor-type forest interactions [33,34], which can be written in the form of $\tau_{\text {forest }}^{i}=\alpha \mu b \sqrt{\rho}$, where $\rho$ is mobile dislocation density and $\alpha$ is a constant. (iii) An external stress linearly increasing with time applied to the simulation region normal to the radial direction. The resultant force decomposed along the slip direction $\tau_{i}$ can be calculated, and the velocity of a mobile dislocation $v_{i}$ can be worked out by the simplified law $v=k \tau$.

Sources resembling the Frank-Read type are randomly distributed in the simulation region with a given density $\rho_{F R S}$. Each of them may probably nucleate a dislocation dipole when subjected to a resultant stress larger than a critical value $\sigma_{n u c}$ continuously over $50 \mathrm{~ms}$. The value of $\sigma_{n u c}$ for these nucleation sources follows a Gaussian distribution with mean stress of $\sim 15 \mathrm{MPa}$ and standard deviation of $\sim 6 \mathrm{MPa}$. The spacing of the nucleated dislocation dipole is $L_{n u c}=\frac{1}{2 \pi} \frac{\mu b}{\sigma_{n u c}}$, so that the nucleated dipole cannot annihilate immediately by its own attraction force. Each dislocation source can only operate once until it has relaxed for an enough time of $250 \mathrm{~ms}$. Precipitate particles are randomly placed in the simulation region. The interactions between dislocations and precipitates are various and complex[35] due to the different precipitate geometries and physical/chemical properties; therefore for simplicity we only consider particle shearing and cross slip in this work. The distance between two neighboring precipitates on a slip plane is supposed to obey a Gaussian distribution, with average 
spacing $l_{\text {ave }}$ and standard deviation $0.3 l_{\text {ave }}$, so that the probability of meeting an obstacle when a dislocation has freely moved a distance $d$ can be calculated as the accumulated integral of the Gaussian distribution. Once a dislocation meets a precipitate, if the resultant stress along the glide plane $\tau_{i}$ is larger than the critical shear stress $\sigma_{c}$, then the dislocation can overcome the precipitate and keeps on moving in the slip plane in a slower speed of $v_{i}=k\left(\tau_{i}-\sigma_{c}\right)$. Otherwise, if the resultant stress normal to the slip direction is larger than another critical stress $\sigma_{c r s}\left(\sigma_{c r s}=3 \sigma_{c}\right)$, the dislocation may have a chance (of $10 \%$ ) to cross slip over the particle. If the above conditions are not met, then the dislocation is considered to be pinned up by the precipitate.

In each time step, a dislocation would move a distance of $d_{i}=v_{i} \times \Delta t$, and so the total strain in this time step can be worked out according to the Orowan equation as $\Delta \varepsilon=\rho b \bar{v} \times \Delta t=$ $(b / A) \sum_{i=1}^{N_{a}} v_{i} \times \Delta t$, where $A$ is the area of the simulation region, and $N_{a}$ is the total number of active dislocations in the current time step. In the simulation, the value $k=0.72 \mu \mathrm{ms}^{-1}(\mathrm{MPa})^{-1}$ in the velocity law is assumed, the time step $\Delta \mathrm{t}$ is set as $1 \mathrm{~ms}$ and the loading rate $\dot{\sigma}$ is $\sim 20 \mathrm{MPas}^{-1}$. The width of the simulation region is varied from $1.5 \mu \mathrm{m}$ to $\sim 6.5 \mu \mathrm{m}$, while the lengths are maintained as three times of the width. The average spacing between precipitates $l_{\text {ave }}$ as well as the critical resistance by precipitates $\sigma_{c}$ are varied to study their effects on the stress-strain behavior. The initial dislocation density $\rho_{0}$ and dislocation source density $\rho_{F R S}$ for pure Al micro-pillars are respectively assumed to be $\sim 2.2 \times 10^{12} \mathrm{~m}^{-2}$ and $\sim 1.1 \times 10^{12} \mathrm{~m}^{-2}$, while for the duralumin case, both $\rho_{0}$ and $\rho_{F R S}$ are increased to two times higher $\sim 4.4 \times 10^{12} \mathrm{~m}^{-2}$ and $\sim 2.2 \times 10^{12} \mathrm{~m}^{-2}$, as the presence of precipitates may introduce more defects into the matrix.

Figure 1(a) shows typical simulated stress-strain curves of pure aluminum pillars with varied size, where the diameters of the micro-pillars are distinguished by different colors. These results are broadly in accordance with experimental observation [8,21], namely, the strength and deformation curves depend on the size of the specimen - the smallest $1.5 \mu \mathrm{m}$ pillars exhibit the steepest stress-strain curves with successive fluctuations and largest scatter between different simulation runs, while the largest $6.5 \mu \mathrm{m}$ pillars show the softest and smoothest stress-strain behavior, with less scattering as well. The simulated $2 \%$ proof strength of pure aluminum micropillars is found to approximately obey a power-law size dependence as shown in Figure 1(b). The simulation data are very close to the experimental observation, and the exponent $m$ in the 
power-law relation $\sigma_{2 \%} \propto D^{-m}$ is about $m=0.79 \pm 0.07$, while experiment data [21] show $m=0.89 \pm 0.07$.

Figure 2(a,b) show the simulated stress-strain behaviors of duralumin micro-pillars, when average spacing of precipitate particles $l_{\text {ave }}$, is $3 \mathrm{~nm}$ and $10 \mathrm{~nm}$ respectively, and in both cases the critical shear stress $\sigma_{c}$ is $51.8 \mathrm{MPa}$. Both groups exhibit much higher strength than the pure $\mathrm{Al}$ case, and an inverse relation between size and strength is still very significant. The stress-strain curves with $l_{\text {ave }}=3 \mathrm{~nm}$ (Figure 2(a)) show that when the pillar size is larger than $3.5 \mu \mathrm{m}$, the three stress-strain curves of the same size on repeated simulation trials are relatively smooth and coincident with very little scattering, while the curves for smaller sizes are much scattered and fluctuating. When $l_{\text {ave }}$ is increased to $10 \mathrm{~nm}$, Figure 2(b), the stress-strain curves for pillars with diameter of $1.5 \mu \mathrm{m}-6.5 \mu \mathrm{m}$ are all jerky with occasional strain bursts occurring even for pillar size of $6.5 \mu \mathrm{m}$. It is suggested that as the precipitate spacing decreases, mobile dislocations in a big pillar has higher chance to meet precipitates which may resist and slow down dislocations before they glide to free surfaces, so that their deformation curves are smoother and more stable. Figure 2(c) shows the relation between the pillar strength and precipitate spacing. As $l_{\text {ave }}$ varies from $3 \mathrm{~nm}$ to $20 \mathrm{~nm}$, the $2 \%$ proof strength of pillars of the same size does not exhibit any significant correlation with $l_{\text {ave }}$, but as $l_{\text {ave }}$ increases beyond $30 \mathrm{~nm}$, small pillars 1.5-2.0 $\mu \mathrm{m}$ big can show much higher strength on increasing $l_{\text {ave }}$, while pillars larger than $3.5 \mu \mathrm{m}$ do not exhibit such a trend and their strength almost remains at the same value, revealing that small sized specimens are more sensitive to a change of the intrinsic structure and the sizedependence of strength may be intensified as the internal length scale increases.

Figure 3 shows the simulated $2 \%$ proof strength $\sigma_{2 \%}$ versus diameter of pillars with precipitates of different critical shear stress $\sigma_{c}$ when spacing $l_{\text {ave }}$ is a constant of $3 \mathrm{~nm}$, together with the experimental data for peak-aged duralumin case [23]. It is obvious that the strength of duralumin micro-pillars exhibits a positive correlation with $\sigma_{c}$, and that their $\sigma_{2 \%}$ still shows power-law scaling with diameter $D$ as $\sigma_{2 \%} \propto D^{-m}$, where $m$ is $0.37 \pm 0.04$ and $0.51 \pm 0.04$ respectively for $\sigma_{c}$ equal $25.9 \mathrm{MPa}$ and $51.8 \mathrm{MPa}$, indicating that the size dependence of strength is weakened with presence of precipitate particles. It is also evident that the value of $\sigma_{c}=$ 51.8MPa can fit the experimental data very well. 
Figure $4(a, b)$ show the variation of the dislocation density inside the specimen with the applied stress. For both the pure $\mathrm{Al}$ and duralumin micro-pillars of diameter $6.5 \mu \mathrm{m}$, their dislocation density increases with the applied stress initially to a plateau and then decreases with further increase of stress, and the pure Al pillars exhibit the lowest dislocation density compared with the ones with precipitates, which agree well with experimental observations [23]. Although pillars with different precipitates show varied peak values of dislocation density, the plateau is very close to the point when the plastic strain starts to rapidly increase with stress as shown in Figures 1(a) and 2(a,b). The results here show that initially dislocations can accumulate and multiply quickly in the specimen with increasing applied stress, and the precipitates may resist the dislocations from moving, until the applied stress is large enough to overcome the critical shear stress of the precipitates $\sigma_{c}$. The dislocations will then keep on gliding and deplete at free surfaces, so that the number of dislocations in the specimen will decrease, even strain bursts will occur on the stress-strain curve. Figure 4(a) also reveals that pillars with precipitates of large $\sigma_{c}$ and dense spacing can show high yield strength and peak value of dislocation density, because in this case the precipitates are more capable and have high probability to resist the mobile dislocations, making them stay inside the confined volume. For the $1.5 \mu \mathrm{m}$ case shown in Figure 4(b), the dislocation density versus stress curves fluctuate rather severely but still exhibit the above-mentioned trend except for the case of pure Al pillars due to the dramatic fluctuations. Because these pillars are so small, even the nucleation or annihilation of only one dislocation would significantly change the average number of active dislocations in the specimen.

This simulation study shows that duralumin micro-pillars exhibit much higher strength than pure Al micro-pillars, while their size dependence of strength is much milder, and all these are comparable to our previous experimental observations [23]. The strength as well as spacing of the precipitates can significantly affect the stress-strain behavior of the micro-pillars. Figure 2 shows that when the precipitate spacing $l_{\text {ave }}$ rises from 3 to $20 \mathrm{~nm}$, the pillar strength does not vary significantly, but the stress-strain curves of big pillars $\sim 5-6 \mu \mathrm{m}$ in size become rough and jerky. As the precipitate spacing $l_{\text {ave }}$ increases to $\sim 30-40 \mathrm{~nm}$, small pillars $\sim 1-2 \mu \mathrm{m}$ in diameter exhibit even higher strength with increasing $l_{\text {ave }}$ but the bigger pillars do not show any significant change; as a result, the exponent $m$ in the scaling law $\sigma_{2 \%} \propto D^{-m}$ becomes larger as $l_{\text {ave }}$ is increased to $\sim 30-40 \mathrm{~nm}$. Previous experiment results [23] show a similar behavior, 
namely, duralumin pillars aged at the peak condition in which the precipitates are larger but more sparsely distributed are found to exhibit a stronger dependence of strength on size, compared with pillars aged at room temperature where the precipitates are smaller in both size and spacing. It is suggested that in a very small crystal, as the precipitate spacing increases, a mobile dislocation may encounter only a few obstacles so that it has a high chance to glide to a free surface, leaving the confined volume in a starved state as shown in Figure 4(b), where the dislocation number decreases significantly when the applied stress is $\sim 150 \mathrm{MPa}$ and plastic strain is only $\sim 1 \%$. The Orowan equation $\dot{\varepsilon}=\rho b v$ suggests that as the dislocation density is decreasing, a higher stress is required to make the limited number of mobile dislocations slip faster so as to maintain a given strain rate. So it is not surprising that small pillars with relatively sparsely distributed precipitates can exhibit even higher strength, and the size dependence of strength can be enhanced.

To conclude, the present simulations show that precipitate hardened duralumin micropillars exhibit power-law size dependent strength, which is mainly determined by the strength and dispersion distance of the precipitates. The fine microstructure can weaken the size dependence of strength, and smooth the stress-strain behavior of duralumin micro-pillars. This work is supported by funding from Kingboard Endowed Professorship in Materials Engineering. 


\section{References}

[1] D.M. Dimiduk, M.D. Uchic, T.A. Parthasarathy, Acta Mater. 53 (2005) 4065.

[2] R. Dou, B. Derby, Scripta Mater. 61 (2009) 524.

[3] J.R. Greer, J.T.M. De Hosson, Prog Mater Sci. 56 (2011) 654.

[4] M.D. Uchic, D.M. Dimiduk, J.N. Florando, W.D. Nix, Science 305 (2004) 986.

[5] D.M. Dimiduk, C. Woodward, R. LeSar, M.D. Uchic, Science 312 (2006) 1188.

[6] J.R. Greer, W.D. Nix, Phys. Rev. B 73 (2006).

[7] D.M. Dimiduk, M.D. Uchic, S.I. Rao, C. Woodward, T.A. Parthasarathy, Modell. Simul. Mater. Sci. Eng. 15 (2007) 135.

[8] K.S. Ng, A.H.W. Ngan, Acta Mater. 56 (2008) 1712.

[9] Z.W. Shan, R.K. Mishra, S.A.S. Asif, O.L. Warren, A.M. Minor, Nat. Mater. 7 (2008) 115.

[10] S.H. Oh, M. Legros, D. Kiener, G. Dehm, Nat. Mater. 8 (2009) 95.

[11] R. Maass, M.D. Uchic, Acta Mater. 60 (2012) 1027.

[12] R. Maass, S. Van Petegem, D. Grolimund, H. Van Swygenhoven, M.D. Uchic, Appl. Phys. Lett. 91 (2007) 131909.

[13] J.R. Greer, W.C. Oliver, W.D. Nix, Acta Mater. 53 (2005) 1821.

[14] T.A. Parthasarathy, S.I. Rao, D.M. Dimiduk, M.D. Uchic, D.R. Trinkle, Scripta Mater. 56 (2007) 313.

[15] S.I. Rao, D.M. Dimiduk, M. Tang, T.A. Parthasarathy, M.D. Uchic, C. Woodward, Philos. Mag. 87 (2007) 4777.

[16] D.M. Norfleet, D.M. Dimiduk, S.J. Polasik, M.D. Uchic, M.J. Mills, Acta Mater. 56 (2008) 2988.

[17] S. Rao, D. Dimiduk, T. Parthasarathy, M. Uchic, M. Tang, C. Woodward, Acta Mater. 56 (2008) 3245.

[18] R. Gu, A.H.W. Ngan, J. Mech. Phys. Solids 61 (2013) 1531.

[19] K.S. Ng, A.H.W. Ngan, Acta Mater. 57 (2009) 4902.

[20] A.T. Jennings, C. Gross, F. Greer, Z.H. Aitken, S.W. Lee, C.R. Weinberger, J.R. Greer, Acta Mater. 60 (2012) 3444.

[21] R. Gu, A.H.W. Ngan, Acta Mater. 60 (2012) 6102.

[22] D. Jang, C. Cai, J.R. Greer, Nano Lett. 11 (2011) 1743.

[23] R. Gu, A.H.W. Ngan, Scripta Mater. 68 (2013) 861. 
[24] K.S. Ng, A.H.W. Ngan, Philos. Mag. 89 (2009) 3013.

[25] A. Kunz, S. Pathak, J.R. Greer, Acta Mater. 59 (2011) 4416.

[26] A. Rinaldi, P. Peralta, C. Friesen, K. Sieradzki, Acta Mater. 56 (2008) 511.

[27] X.W. Gu, C.N. Loynachan, Z. Wu, Y.-W. Zhang, D.J. Srolovitz, J.R. Greer, Nano Lett. 12 (2012) 6385.

[28] K.A. Afanasyev, F. Sansoz, Nano Lett. 7 (2007) 2056.

[29] D. Jang, X. Li, H. Gao, J.R. Greer, Nat. Nanotechnol. 7 (2012) 594.

[30] L. Nicola, E. Van der Giessen, A. Needleman, J. Appl. Phys. 93 (2003) 5920.

[31] Z. Li, C. Hou, M. Huang, C. Ouyang, Comput. Mater. Sci. 46 (2009) 1124.

[32] R. Kumar, L. Nicola, E. Van der Giessen, Mater. Sci. Eng. A 527 (2009) 7.

[33] P.S.S. Leung, N. A.H.W, Scripta Mater. 69 (2013) 235.

[34] A.A. Benzerga, J. Mech. Phys. Solids 57 (2009) 1459.

[35] J.W. Martin, Precipitation Hardening, Butterworth-Heinemann, Boston,1998. 


\section{Figure Captions:}

Figure 1 - (a) Simulated stress-strain behaviors of pure Al micro-pillars, and their (b) $2 \%$ proof strength versus diameter relation, in comparison with experimental data [21].

Figure 2 - Simulated stress-strain behaviors of duralumin micro-pillars with mean precipitate spacing of (a) $3 \mathrm{~nm}$ and (b) $10 \mathrm{~nm}$, and (c) their $2 \%$ proof strength with varying precipitate spacing.

Figure $3-2 \%$ proof strength versus diameter of duralumin micro-pillars with mean precipitate spacing of $3 \mathrm{~nm}$, in comparison with experimental data [23].

Figure 4 - Dislocation density of duralumin (a) $6.5 \mu \mathrm{m}$ and (b) $1.5 \mu \mathrm{m}$ pillars varied with applied stress in the simulation. 


\section{Figures}

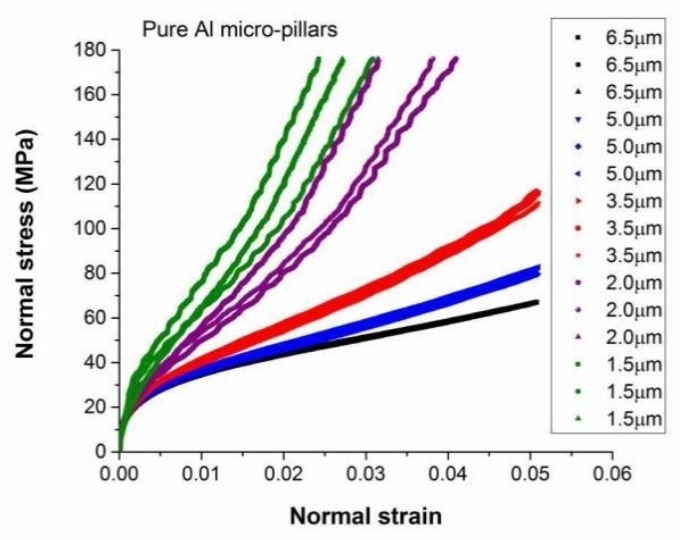

(a)

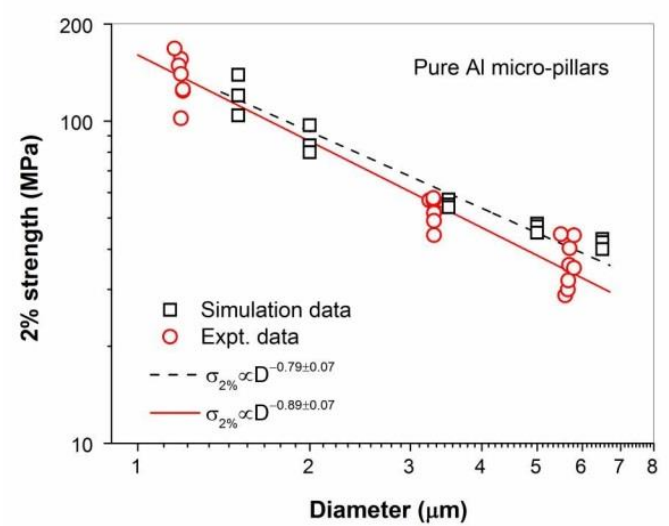

(b)

Figure 1 - (a) Simulated stress-strain behaviors of pure Al micro-pillars, and their (b) 2\% proof strength versus diameter relation, in comparison with experimental data [21]. 


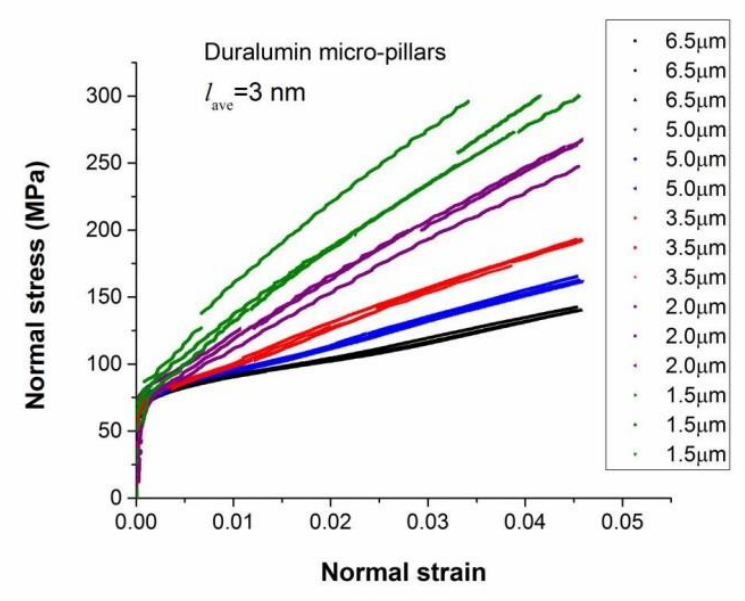

(a)

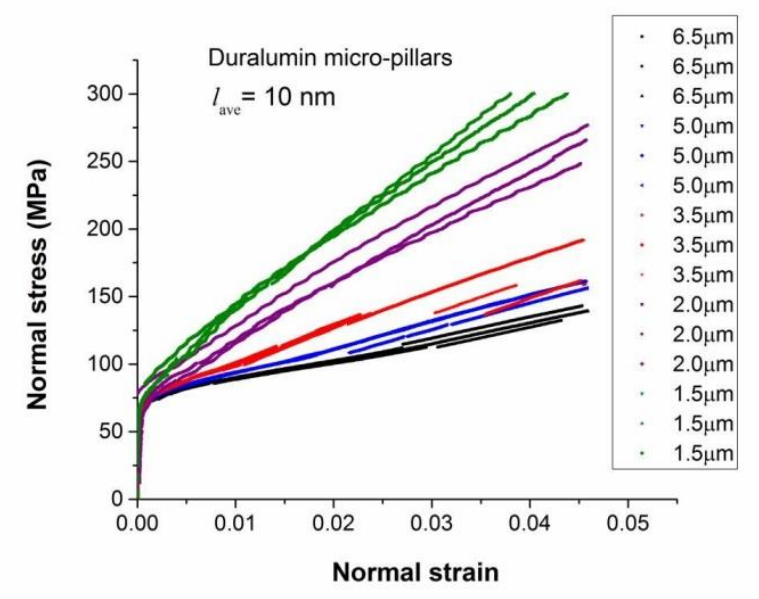

(b)

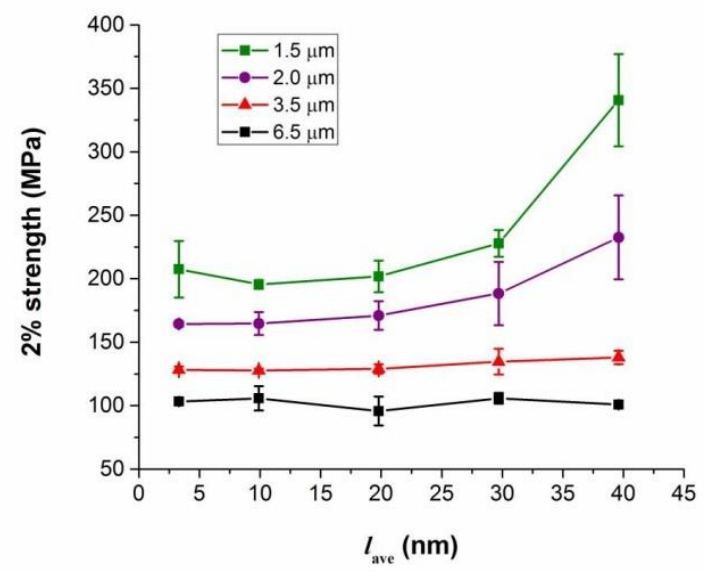

(c)

Figure 2 - Simulated stress-strain behaviors of duralumin micro-pillars with mean precipitate spacing of (a) $3 \mathrm{~nm}$ and (b) $10 \mathrm{~nm}$, and (c) their $2 \%$ proof strength with varying precipitate spacing. 


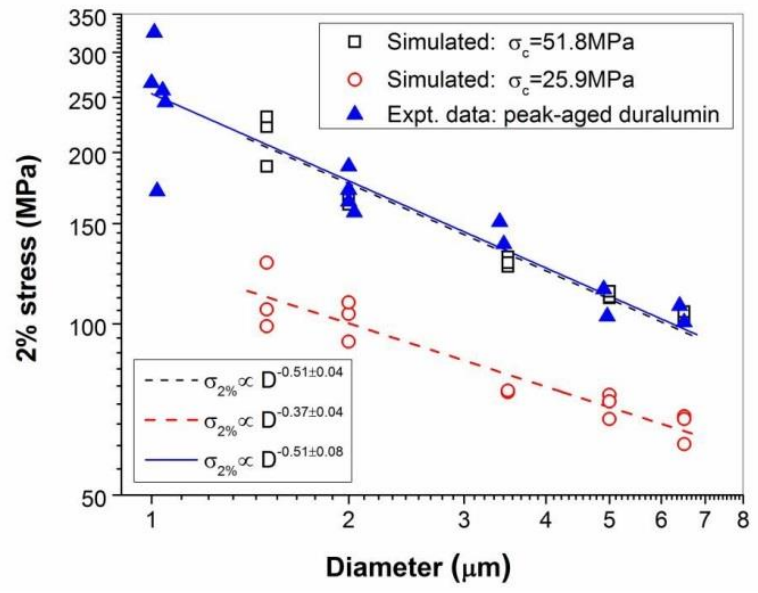

Figure $3-2 \%$ proof strength versus diameter of duralumin micro-pillars with mean precipitate spacing of $3 \mathrm{~nm}$, in comparison with experimental data [23].
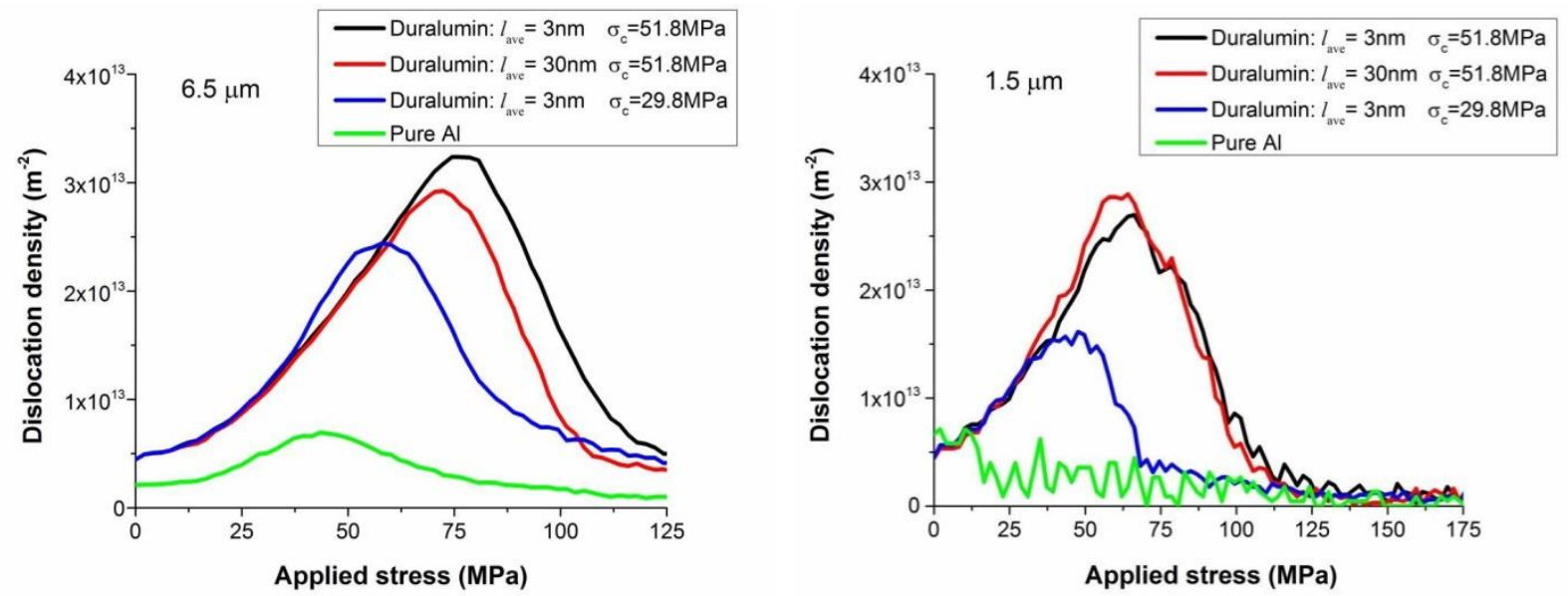

Figure 4 - Dislocation density of duralumin (a) $6.5 \mu \mathrm{m}$ and (b) $1.5 \mu \mathrm{m}$ pillars varied with applied stress in the simulation. 